\title{
AV Fistula Stenosis
}

National Cancer Institute

\section{Source}

National Cancer Institute. AV Fistula Stenosis. NCI Thesaurus. Code C114754.

Narrowing of the lumen of an arteriovenous fistula. 